\title{
Papuamine Inhibits Viability of Non-small Cell Lung Cancer Cells by Inducing Mitochondrial Dysfunction
}

\author{
HYE-YOUNG MIN ${ }^{1}$, YUJIN JUNG ${ }^{2}$, KWAN HEE PARK ${ }^{3}$ and HO-YOUNG LEE ${ }^{1,2,3}$ \\ ${ }^{1}$ Department of Molecular Medicine and Biopharmaceutical Sciences, \\ Graduate School of Convergence Science and Technology, and College of Pharmacy, \\ Seoul National University, Seoul, Republic of Korea; \\ ${ }^{2}$ Interdisciplinary Program in Genetic Engineering, Seoul National University, Seoul, Republic of Korea; \\ ${ }^{3}$ College of Pharmacy and Research Institute of Pharmaceutical Sciences, \\ Seoul National University, Seoul, Republic of Korea
}

\begin{abstract}
Background/Aim: Despite the Warburg effect, mitochondria play an essential role in the survival and maintenance of cancer cells. Thus, mitochondria have been considered a target for anticancer agents. Here, we identified a mitochondria-targeting anticancer agent from natural products. Materials and Methods: Morphological and functional changes in mitochondria were determined by a fluorescence-based High Content Imaging System. Using human non-small cell lung cancer (NSCLC) cell lines (H1299, $H 226 B$, and A549), cell viability and colony formation assays, cell cycle analysis, and immunoblotting were performed to determine cytotoxic and proapoptotic effects of papuamine. Results: Using a natural product chemical library, we identified papuamine as an active compound to inhibit viability and ATP production of NSCLC cells. Papuamine depleted intracellular ATP by causing mitochondrial dysfunction, as indicated by the loss of the mitochondrial membrane potential and increased mitochondrial superoxide generation. Papuamine significantly inhibited viability and colony formation of NSCLC cells by inducing apoptosis. Conclusion: Papuamine has a potential as a novel mitochondria-targeting anticancer agent.
\end{abstract}

Despite extensive efforts to develop effective therapies, lung cancer is still the leading cause of cancer-related death worldwide. The 5-year survival rate for lung cancer is about $20 \%(1,2)$. Recently developed anticancer agents targeting $E G F R$ mutations or abnormal $A L K$ fusions benefit a subset

Correspondence to: Ho-Young Lee, Ph.D., College of Pharmacy and Research Institute of Pharmaceutical Sciences, Seoul National University, Seoul 08826, Republic of Korea. Tel: +82 28809277, Fax: +82 262805327, e-mail: hylee135@snu.ac.kr

Key Words: Papuamine, alkaloid, anticancer, mitochondria. of patients with non-small cell lung cancer (NSCLC), which accounts for approximately $85 \%$ of lung cancer cases (3). These drugs are now applied as a first-line therapy (4). In addition, immune checkpoint inhibitors targeting programmed cell death-1 (PD-1) or programmed cell deathligand 1 (PD-L1) have beneficial effects on the survival of patients with advanced NSCLC and have recently received approval for clinical use (5). However, drug resistance and tumor recurrence inevitably emerge with these therapies, eventually leading to poor clinical outcomes $(6,7)$. Thus, there is an urgent need to develop alternative strategies to treat NSCLC.

Cancer cells require high levels of energy production for their survival and proliferation, and deregulated energy metabolism has been regarded as a hallmark of cancer (8). Mitochondria play an important role in the maintenance of cellular homeostasis and are crucial regulators of cell survival through their ability to modulate ATP production and apoptosis (9). Although cancer cells achieve high levels of energy production via metabolic reprogramming and mitochondrial dysfunction, the mitochondria in cancer cells are still functional and play a crucial role in cell survival (10). Therefore, mitochondria may be considered a target for anticancer therapy. Several compounds have already been identified as anticancer agents targeting mitochondria (9). However, the anticancer effects of these mitochondriatargeting drugs have been minimal, and their applications have been limited as a result of low efficacy and severe toxicity. For example, glycolysis inhibitors, such as 2deoxyglucose and 3-bromopyruvate, are only marginally effective as single therapies (11). In addition, despite their anticancer effects, the clinical use of mitochondrial complex I inhibitors, such as phenformin and rotenone, is limited due to severe side effects, including lactic acidosis and Parkinson's-like symptoms, respectively $(12,13)$. Thus, it is necessary to identify novel mitochondria-targeting anticancer 
drugs with different structures and/or mechanisms of action to develop effective anticancer therapeutic strategies.

Papuamine is a pentacyclic alkaloid derived from marine sponges, such as Haliclona sp. (14). Several previous findings have suggested that papuamine possesses antifungal activity (14), induces autophagy (15), modulates epithelialmesenchymal transition (16), and displays synergistic anticancer effects with doxorubicin (17). The extract of Haliclona $s p$., which contains papuamine as an active principle, inhibits the viability of NSCLC cells via inducing apoptosis (18). A previous report also demonstrated that papuamine rapidly induces mitochondrial dysfunction, followed by the induction of autophagy, the upregulation of JNK activation, and, eventually, apoptotic cell death (19). However, additional antitumor mechanisms of action or other biological properties of papuamine still remain to be elucidated.

Here, we identified the pentacyclic alkaloid papuamine as a novel mitochondria-targeting anticancer agent. Papuamine reduced cellular ATP levels in a time- and dose-dependent manner. Consistently, papuamine induced a dose-dependent activation of AMP activated kinase (AMPK), resulting in the downregulation of signaling pathways responsible for cell growth, such as those functioning through mammalian target of rapamycin (mTOR) and p70S6K. Further studies demonstrate that papuamine rapidly induced mitochondrial dysfunction as indicated by mitochondrial fission, decreased mitochondrial mass, mitochondrial membrane depolarization, and mitochondrial superoxide over-production and increased cellular oxidative stress. In addition, papuamine significantly inhibited NSCLC cell viability, induced apoptosis, and reduced colony formation in both anchorage-dependent and -independent conditions. These results collectively suggest the potential of papuamine as a novel mitochondria-targeting anticancer agent for the treatment of NSCLC.

\section{Materials and Methods}

Chemicals and reagents. Mouse monoclonal antibodies against cleaved poly (ADP-ribose) polymerase (PARP) were purchased from BD Biosciences (San Jose, CA, USA). Rabbit polyclonal antibodies against pAMPK (T172), AMPK, pmTOR (S2448), mTOR, p70S6K (T389), and p70S6K were purchased from Cell Signaling Technology (Danvers, MA, USA). Primary antibodies against actin and the corresponding horseradish peroxidase (HRP)conjugated secondary antibodies were purchased from Santa Cruz Biotechnology (Santa Cruz, CA, USA). 3-(4,5-dimethylthiazol-2yl)-2,5-diphenyl tetrazolium bromide (MTT), propidium iodide (PI), and other chemicals were purchased from Sigma-Aldrich (St. Louis, MO, USA) unless otherwise specified.

Test compounds. We used a repositioned chemical library consisting of various compounds representative of each chemical class of natural products-derived compounds, including alkaloids, flavonoids, tannins, lignans, and terpenoids. These chemicals are
Table I. List of compounds used in this study.

\begin{tabular}{lc}
\hline Compound & Name \\
\hline 1 & Neorautenol \\
2 & Rutin \\
3 & Puerarin \\
4 & Schizandrin \\
5 & Magnolol \\
6 & Curcumin \\
7 & Amuresin G \\
8 & Epigallocatechin gallate \\
9 & Decursinol \\
10 & Oxymatrine \\
11 & Aloin \\
12 & Harmaline \\
13 & Papuamine \\
14 & Boldine \\
15 & (+)-Bicuculline \\
16 & Sinomenine \\
17 & Scopolamine \\
18 & Vincamine \\
19 & Noscarpine \\
20 & Crotaline \\
21 & Reserpine \\
22 & Dihydrocapsaicin \\
23 & Oxymatrine \\
24 & Quinone \\
25 & Alpha-solanine \\
\hline
\end{tabular}

listed in Table I. Papuamine was purchased from Enzo Life Sciences (Farmingdale, NY, USA).

Cell culture. Human NSCLC cell lines (H1299, H226B, and A549) were purchased from American Type Culture Collection (ATCC, Manassas, VA, USA) or kindly provided by Dr. John V. Heymach (MD Anderson Cancer Center, Houston, TX, USA). Cells were cultured in RPMI 1640 medium supplemented with $10 \%$ fetal bovine serum (FBS) and antibiotics (all from Welgene Inc., Gyeongssan-si, Republic of Korea). Cells were maintained at $37^{\circ} \mathrm{C}$ with $5 \% \mathrm{CO}_{2}$ in a humidified atmosphere.

MTT assay. Cells were seeded into 96-well plates at a density of $1 \times 10^{3}$ cells/well and incubated for $24 \mathrm{~h}$. The cells were then treated with vehicle or papuamine diluted in complete media for three days. Next, the cells were treated with the MTT solution (at a final concentration of $500 \mu \mathrm{g} / \mathrm{ml}$ ) and incubated for two $\mathrm{h}$ at $37^{\circ} \mathrm{C}$. The formazan products were dissolved in DMSO, and the absorbance was measured at $570 \mathrm{~nm}$. The data are presented as a percentage of the control group.

Crystal violet assay. Cells were seeded into 96-well plates at a density of $2 \times 10^{3}$ cells/well and incubated for 1 day. The cells were treated with vehicle or papuamine diluted in complete media for three days. After incubation, cells were fixed with $100 \%$ methanol. Fixed cells were stained with $0.01 \%$ crystal violet solution and washed several times with tap water. Stained cells were dissolved in $100 \%$ methanol, and the absorbance was measured at $570 \mathrm{~nm}$. The data are presented as a percentage of the control group. 
Anchorage-dependent and -independent colony formation assays. For anchorage-dependent colony formation assays, cells were seeded into 6-well plates at a density of 300 cells/well and treated with various concentrations of papuamine diluted in complete medium. The cells were treated for 14 days, and the medium was changed every three days. At the end of the experiment, cells were fixed with $100 \%$ methanol for $15 \mathrm{~min}$ and stained with $0.01 \%$ crystal violet. For anchorage-independent colony formation assays, cells (500 cells/well) were mixed with a sterile 1\% agar solution (final concentration of $0.4 \%$ ) and poured onto a solidified bottom layer of agar (final concentration of $1 \%$ ) in 24-well plates. Papuamine diluted in complete medium was added to the solidified agar, and the medium was changed every three days. Colonies were grown for 10-14 days and then stained with the MTT solution. Colonies were counted using ImageJ software (National Institute of Health, Bethesda, MD, USA) (20).

Determination of ATP production. Intracellular ATP levels were determined using ATPlite ${ }^{\mathrm{TM}}$ (PerkinElmer, Waltham, MA, USA) according to the manufacturer's recommended procedure. Cells were seeded into black, clear-bottom 96-well plates (Corning, Corning, NY, USA) at a density of 1,000 cells/well and incubated for $24 \mathrm{~h}$. Cells were treated with test compounds for six hours and then lysed with lysis buffer. Lysates were incubated with the ATPlite substrate, and luminescence was then measured on a SpectraMAX M5 microplate reader (Molecular Devices, Sunnyvale, CA, USA).

Fluorescence imaging analysis. Cells were seeded onto round coverslips in 12-well plates and allowed to attach overnight. The cells were treated with papuamine for the indicated time periods and then stained with the indicated fluorescence dye [100 nM Mitotracker, $100 \mathrm{nM}$ TMRM, $5 \mu \mathrm{M}$ Mitosox, or $30 \mu \mathrm{M}$ 2-NBDG (all from Thermo Fisher Scientific, Waltham, MA, USA)] for 15 (for Mitosox) or $30 \mathrm{~min}$ (for others). For Mitosox staining, cells were counterstained with Hoechst $33342(1 \mu \mathrm{g} / \mathrm{ml})$. The cells were washed twice with PBS and coverslips were mounted with Vectamount (Vector Laboratories, Burlingame, CA, USA). Fluorescent images were obtained using a Nikon Eclipse 80i microscope (Nikon Corp., Tokyo, Japan) equipped with a Nuance multispectral imaging system (PerkinElmer, Alameda, CA, USA) or analyzed using the Operetta High Content Imaging System (PerkinElmer).

Cell cycle analysis. Cells were treated with increasing concentrations of papuamine for three days. Adherent and floating cells were collected and fixed in ice-cold $100 \%$ methanol overnight at $-20^{\circ} \mathrm{C}$. Cells were stained with a propidium iodide $(50 \mu \mathrm{g} / \mathrm{ml})$ solution containing RNase A $(50 \mu \mathrm{g} / \mathrm{ml})$ for $30 \mu \mathrm{min}$ at room temperature. Apoptotic cells were analyzed by flow cytometry using a FACSCalibur ${ }^{\circledR}$ flow cytometer (BD Biosciences).

Western blot analysis. Cells were harvested with modified RIPA lysis buffer [50 mM Tris- $\mathrm{HCl}(\mathrm{pH} 7.5), 150 \mathrm{mM} \mathrm{NaCl}, 1 \mathrm{mM}$ EDTA, $0.25 \%$ sodium deoxycholate, $1 \%$ Triton X-100, $1 \mathrm{mM}$ $\mathrm{Na}_{3} \mathrm{VO}_{4}, 100 \mathrm{mM} \mathrm{NaF}, 0.5 \mathrm{mM}$ DTT, $1 \mathrm{mM}$ PMSF, $1 \mu \mathrm{g} / \mathrm{ml}$ aprotinin, $1 \mu \mathrm{g} / \mathrm{ml}$ leupeptin, and $20 \mathrm{mM} \beta$-glycerophosphate]. The lysates were centrifuged at $13,000 \mathrm{rpm}$ for $30 \mathrm{~min}$ at $4^{\circ} \mathrm{C}$. Equal amounts of protein were separated by $8-10 \%$ SDS-PAGE and transferred onto PVDF membranes. Membranes were blocked with blocking buffer (3\% BSA in Tris-buffered saline- $0.1 \%$ Tween-20 (TBST) containing $0.02 \%$ sodium azide) for one $\mathrm{h}$ at room temperature. Membranes were then incubated with a primary antibody (1:1000 dilution in blocking buffer) overnight at $4^{\circ} \mathrm{C}$. The membrane was washed four times with TBST for one $h$ at room temperature and then incubated with the corresponding secondary antibody (1:5000 dilution in 3\% skim milk in TBST). Membranes were washed four times with TBST for one $\mathrm{h}$ at room temperature. The blots were visualized using an enhanced chemiluminescence (ECL) detection kit (Thermo Fisher Scientific).

Statistical analyses. The data are presented as the mean \pm SD. All experiments were independently performed at least twice, and a representative result is presented. The data were calculated and analyzed using Microsoft Excel software (Microsoft Corp., Redmond, MA, USA). Statistical significance was determined using a two-sided Student's $t$-test. A $p$-value of less than 0.05 was considered significant.

\section{Results}

Papuamine: an active compound that suppresses cellular energetics on NSCLC cells. Recent studies have suggested the importance of targeting cellular metabolism for anticancer therapy $(21,22)$. Diverse metabolic pathways in cancer cells commonly lead to energy production, and ATP is universally seen as the energy exchange factor (23). Thus, to identify a novel cytotoxic compound targeting cellular energetics in NSCLC cells, we evaluated the ability of various natural product-derived compounds to inhibit the viability and ATP production of H1299 human NSCLC cells. We first examined a natural product chemical library consisting of 13 compounds representing diverse structural classes, such as flavonoids, lignans, and alkaloids (Table I). As shown in Figure 1B (left), compound 13 displayed the most significant inhibitory effects on cell survival and ATP production. Because compound $\mathbf{1 3}$ is the pentacyclic alkaloid papuamine (Figure 1A), we further compared the inhibitory effects of compound $\mathbf{1 3}$ with those of additional alkaloids (Table I). As shown in Figure 1B (right), compound 13 (papuamine) displayed the greatest inhibitory effect on ATP production. Moreover, only compound $\mathbf{1 3}$ showed a significant reduction in H1299 cell viability (Figure 1C). Thus, we identified papuamine as novel anti-cancer candidate to target cellular energetics of NSCLC.

Papuamine decreases cellular ATP levels and upregulates AMPK in NSCLC cells. Based on the ability of papuamine to inhibit cellular ATP production in H1299 cells (Figure 1B), we sought to confirm the effect of papuamine on cellular energy production. As shown in Figure 2A and B, papuamine significantly reduced cellular ATP levels in NSCLC cells in a time- and concentration-dependent manner. The phosphorylation and activation of AMPK, an energy and nutrient sensor that maintains energy homeostasis (24), was consistently elevated in cells treated with papuamine in a time- and dose-dependent manner. Meanwhile, the activation of mTOR and its downstream target p70S6K decreased 

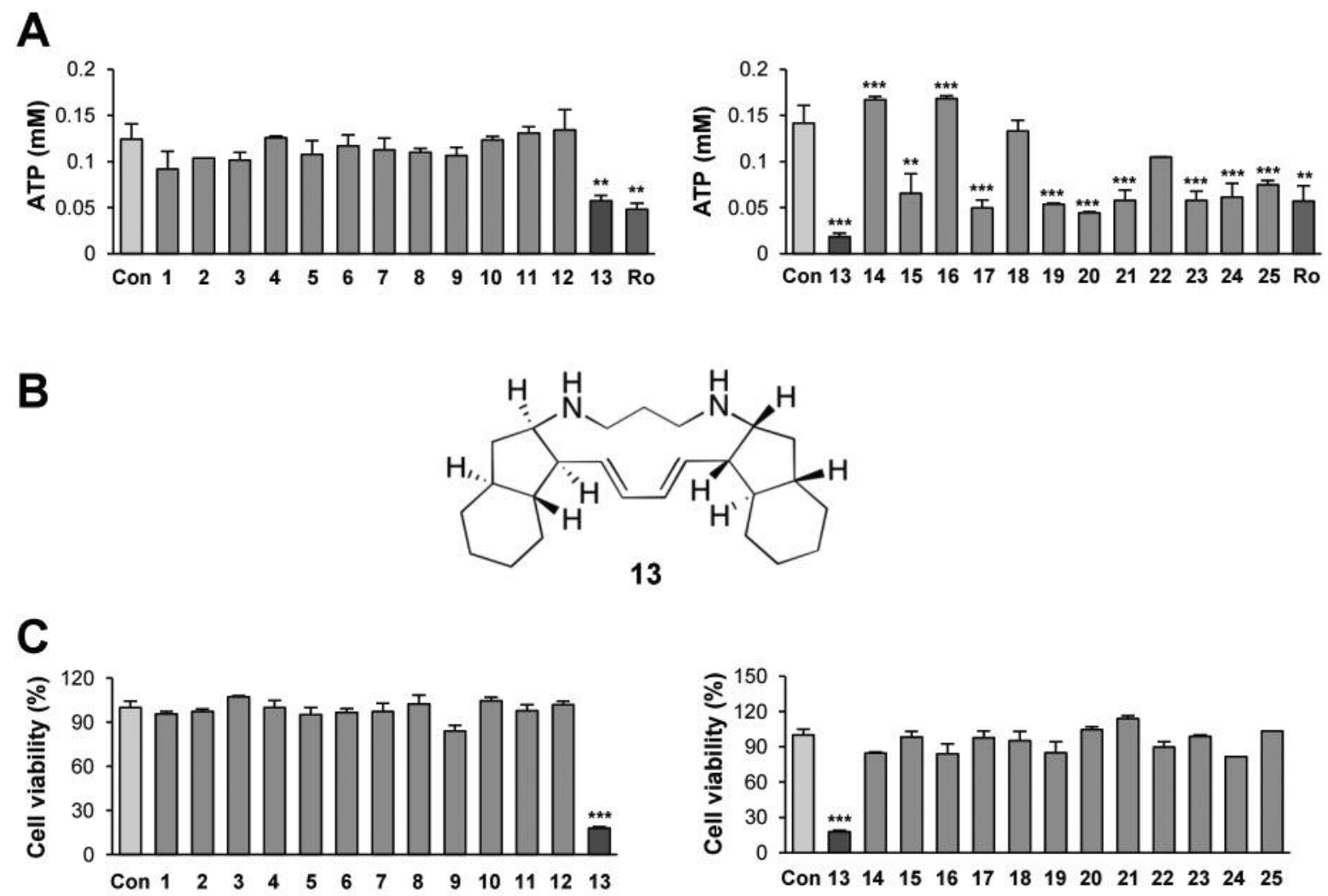

Figure 1. Identification of papuamine as an inhibitor of cellular energetics on NSCLC cell. (A) The effect of several natural products-derived compounds $(10 \mu \mathrm{M})$ on cellular ATP production in $H 1299$ cells was determined using the luciferase-based ATP determination assay. Rotenone (Ro; $1 \mu M$ ) was used as the positive control. (B) The chemical structure of compound 13 (papuamine). (C) The MTT assay was used to evaluate the effect of several natural product-derived compounds $(10 \mu \mathrm{M})$ on the viability of $H 1299$ cells. The bars represent the mean $\pm S D ; * p<0.05$, **p<0.01, and $* * * p<0.001$, as determined by a two-sided Student's $t$-test.

following papuamine treatment (Figure 2C and D). These results indicate that papuamine acts as an AMPK activator by decreasing cellular energy levels in NSCLC cells.

Papuamine causes mitochondrial dysfunction in NSCLC cells. Because papuamine rapidly reduced intracellular ATP and elevated AMPK activation in NSCLC cells, we next examined the effect of papuamine on mitochondrial function. To assess papuamine-induced changes in mitochondrial structure, NSCLC cells were incubated with MitoTracker probes to label mitochondria (25) and then subjected to image-based texture analysis using Operetta. Papuamine elevated the fluorescence intensity in a dose-dependent manner, indicating an increase in the number of mitochondria. However, the number of mitochondria with normal, ridge-like morphology gradually decreased following papuamine treatment (Figure 3A-C). These findings collectively suggest that papuamine leads to mitochondrial fission and generates damaged mitochondria, eventually causing mitochondrial dysfunction. To ensure papuamine-induced mitochondrial deregulation, we utilized the TMRM (26) and Mitosox (27) probes to determine the mitochondrial membrane potential and mitochondrial superoxide levels, respectively. Papuamine markedly reduced mitochondrial membrane potential as indicated by the decrease in the cytosolic fluorescence signal (Figure 3D). Meanwhile, the red fluorescence signal, an indicator of mitochondrial superoxide levels, was time-dependently increased in papuamine-treated cells (Figure 3E).

In addition, the regulation of glucose uptake by papuamine treatment was examined using the fluorescent indicator 2-NBDG (28). As shown in Figure 3F, papuamine had no effect on glucose uptake. These results clearly indicate that papuamine mediates mitochondrial dysfunction.

Papuamine suppresses the cell viability and colony formations in NSCLC cells. Based on the ability of papuamine to induce mitochondrial dysfunction, we evaluated the effect of papuamine, as a novel inhibitor of cellular energetics, on cell viability and colony formations. 



Figure 2. Reduction of cellular ATP production and consequent AMPK activation following papuamine treatment. (A, B) Time-dependent inhibitory effects of $5 \mu M$ papuamine (A) and dose-dependent inhibitory effects of papuamine for $3 h(B)$ on cellular ATP production in NSCLC cells, determined by the luciferase-based ATP determination assay. (C, D) H1299 cells were treated with papuamine (5 $\mu M)$ for the indicated periods $(C)$ or papuamine $(1$ and $5 \mu M)$ for $24 h(D)$. The expression levels of total and phosphorylated AMPK and its downstream signaling proteins were determined by western blot analysis. PA: Papuamine. The bars represent the mean $\pm S D ; * p<0.05, * * p<0.01$, and $* * * p<0.001$, as determined by a two-sided Student's t-test.

Treatment with papuamine inhibited NSCLC viability in a dose-dependent manner (Figure 4A). Because the MTT assay determines cell viability based on the mitochondrial dehydrogenase activity in live cells (29), we confirmed the inhibitory effect of papuamine on the viability of NSCLC cells by a crystal violet assay. Consistent with the MTT assay, papuamine significantly inhibited the viability of H1299 NSCLC cells (Figure 4B). Colony formation under both anchorage-dependent and -independent culture conditions was also significantly suppressed by treatment with papuamine (Figure 4C and D). These results indicate that papuamine is capable of suppressing the viability and colony-forming ability of NSCLC cells.

Papuamine induces apoptotic cell death. Because papuamine exhibits significant inhibitory effects on cell viability and colony formation, we next investigated whether papuamine may induce apoptosis in NSCLC cells. We first assessed morphological changes in cells treated with papuamine using the Operetta High Content Imaging System. As shown in Figure 5A and B, papuamine treatment decreased cell number, cell size, and nuclear area but increased nuclear intensity, indicating cell shrinkage and chromatin condensation, which are features of apoptotic cell death $(30,31)$. Papuamine treatment also increased the number of cells in the sub-G1 phase and the cleavage of poly(ADP-ribose) polymerase (PARP) and caspase 3 (Figure 5C and D), confirming that apoptosis plays a role in the inhibitory effect of papuamine on NSCLC cell viability and colony formations.

\section{Discussion}

In the present study, we identified papuamine as a novel agent to suppress cellular energy production and viability in NSCLC cells. We showed that papuamine triggered mitochondrial dysfunction, thereby inducing mitochondrial 
A

PA $(\mu \mathrm{M})$

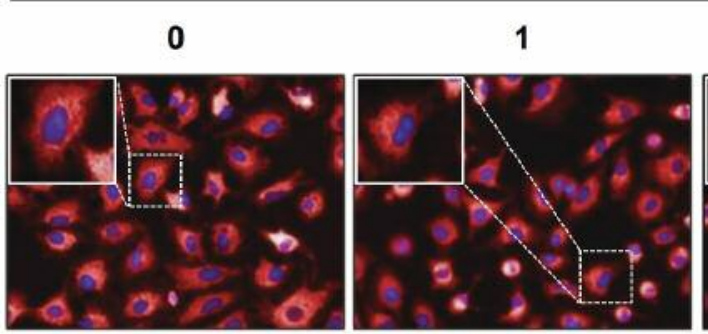

5

10
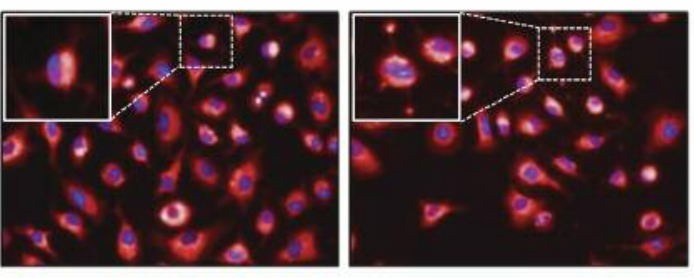

B
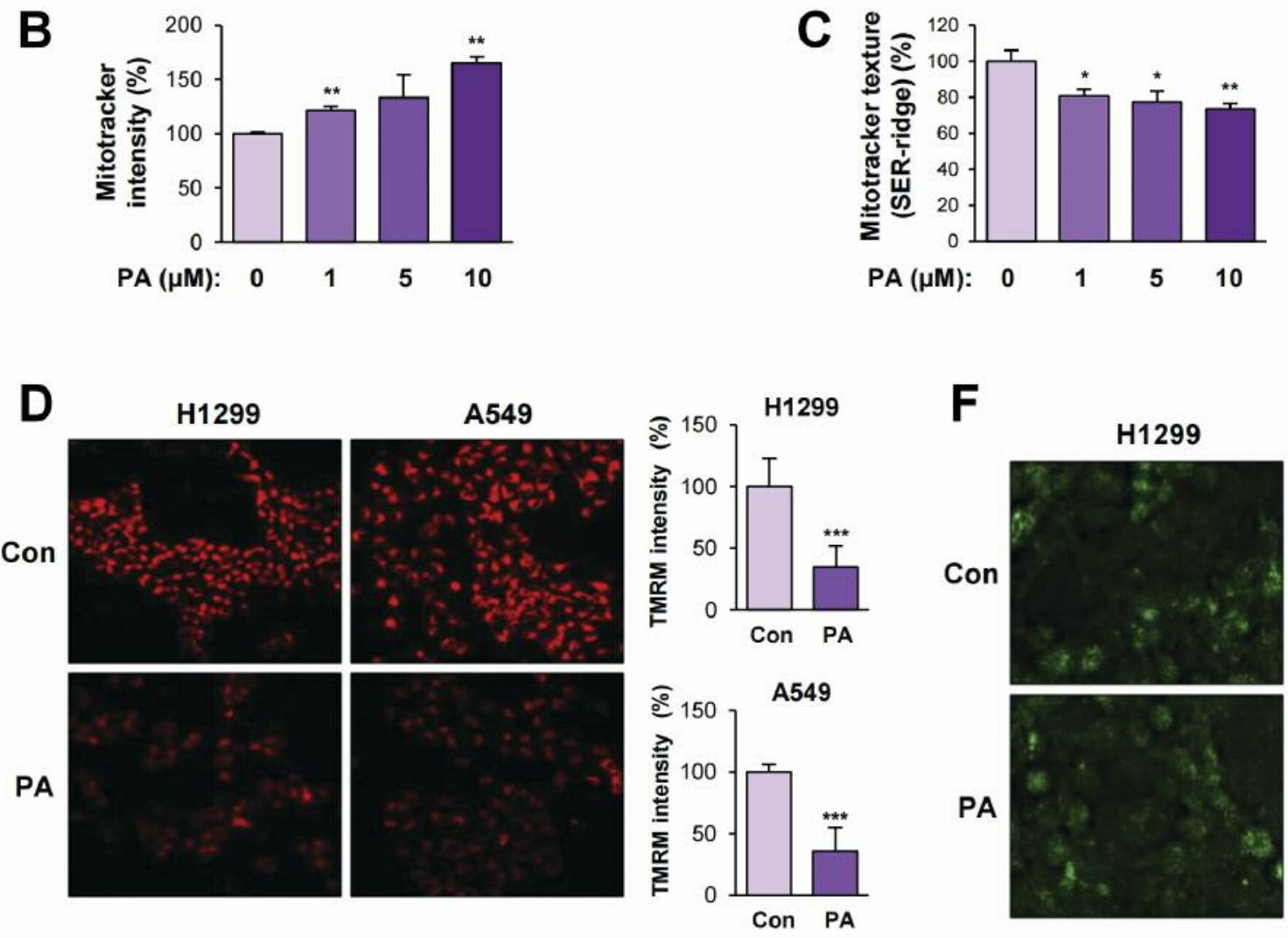

$\mathbf{E}$ Time (h)
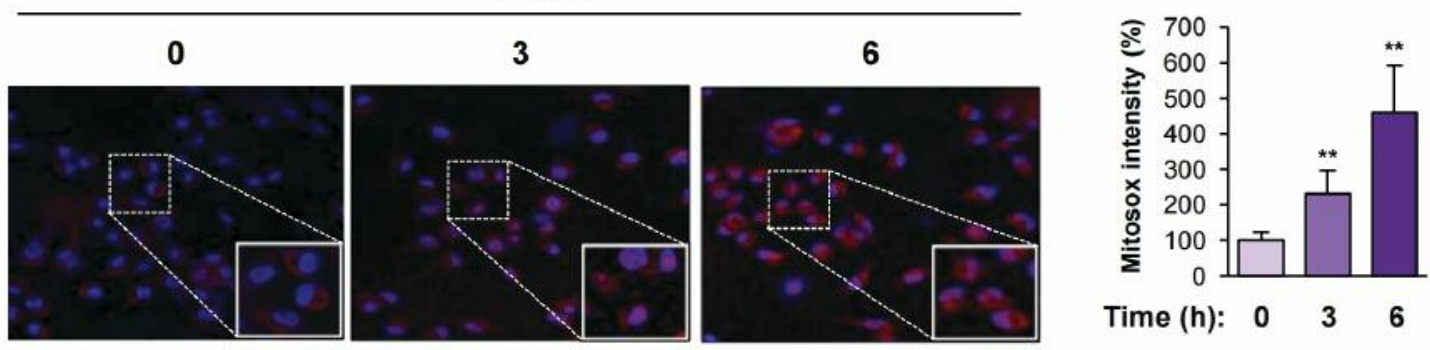

Figure 3. Mitochondrial dysfunction caused by papuamine treatment. (A-F) H1299 cells were treated with various concentrations of papuamine (A$C)$ for $6 \mathrm{~h}$ or papuamine $(5 \mu M)$ for 3 or $6 \mathrm{~h}(D-F)$. The cells were stained with Mitotracker $(A-C)$, TMRM (D), Mitosox $(E)$ or 2-NBDG $(F)$ as described in the Materials and Methods section. The cells were observed under a fluorescence microscope or analyzed by Operetta. (D and E, right) Quantification of the changes of TMRM $(D)$ and Mitsox $(E)$ intensity in each test group. Con: Control; PA: papuamine. The bars represent the mean $\pm S D ; * p<0.05, * * p<0.01$, and ${ }^{* * *} p<0.001$, as determined by a two-sided Student's t-test. 
A

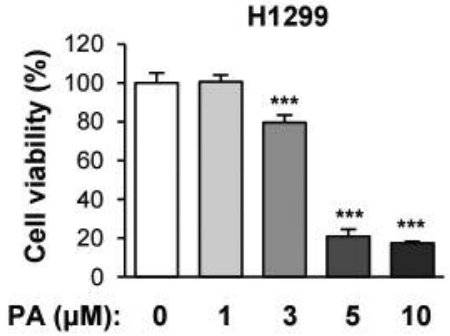

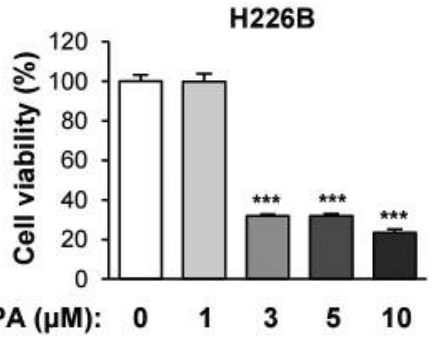

D

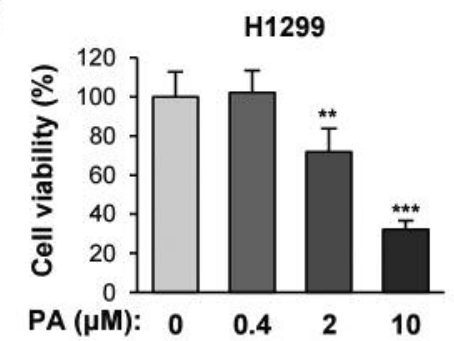

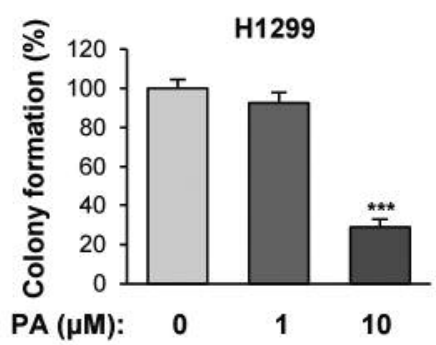

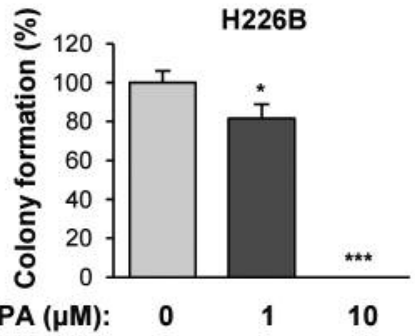

PA $(\mu \mathrm{M}):$

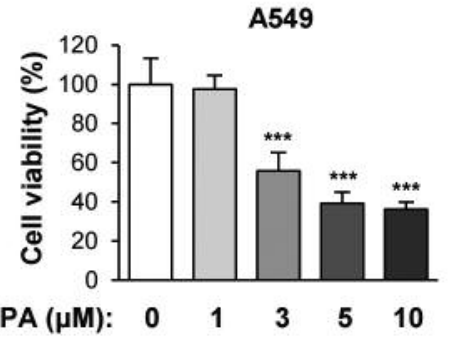

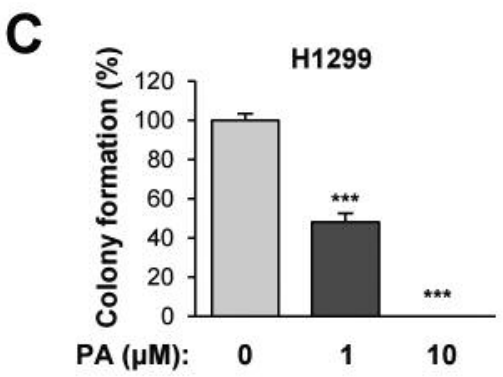

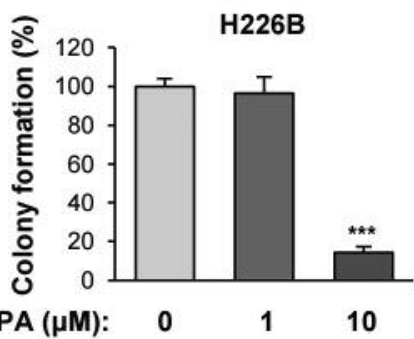

Figure 4. The inhibition of cell viability and colony formations by papuamine treatment. $(A, B) M T T(A)$ and crystal violet $(B)$ assays were used to evaluate the ability of various concentrations of papuamine to inhibit the viability of NSCLC cell lines. (C, D) The ability of various concentrations of papuamine to inhibit colony formation in several NSCLC cell lines under both anchorage-dependent and-independent conditions was determined using the anchorage-dependent colony formation $(C)$ and soft agar $(D)$ assays. Con: Control; PA: papuamine. The bars represent the mean $\pm S D$; ${ }^{*} p<0.05, * * p<0.01$, and $*^{* *} p<0.001$, as determined by a two-sided Student's $t$-test.

fission and ultimately apoptotic cell death. These results indicate the potential of papuamine as a mitochondriatargeting anticancer agent for the treatment of NSCLC.

Despite the development of therapeutic regimens for NSCLC treatment, such as molecular targeted therapy and immunotherapy, drug resistance and limited clinical benefits are still significant obstacles. Thus, the development of alternative therapeutic options is necessary. Considering 1) the metabolic reprogramming in lung cancer $(32), 2$ ) the improvement of anticancer therapeutics by targeting cellular metabolism (33), and 3) the crucial role of mitochondria in the regulation of cellular metabolism and energy production (34), overcoming drug resistance by utilizing mitochondria-targeting anticancer agents may be the key to developing effective anticancer therapeutics (9). Indeed, a previous study identified a novel mitochondria-targeting agent with anticancer activity (35), while strategies for the discovery of novel mitochondriatargeting anticancer compounds (36) and the delivery of anticancer drugs to mitochondria using nanoparticles (37) have also been recently developed. Moreover, although it is known that cancer cells utilize glycolysis as a main energy source, the utilization of glycolysis as an energy source may rely on the cell types, origins, or environments and cancer cells have diverse metabolic pathways for energy production (38). In addition, the contribution of glycolysis to the energy production in cancer cells does not exceed $60 \%(38,39)$ and thus mitochondrial oxidative phosphorylation still plays an important role in bioenergetics in cancer cells (38). 
A
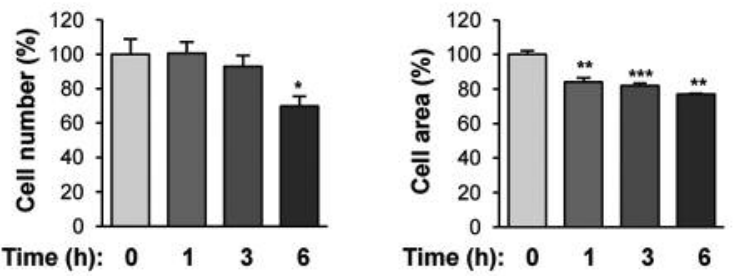

B

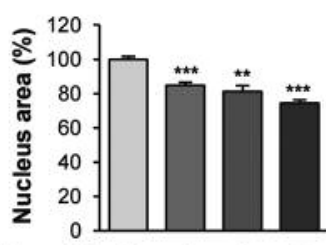

Time (h): $\begin{array}{llll}0 & 1 & 3 & 6\end{array}$

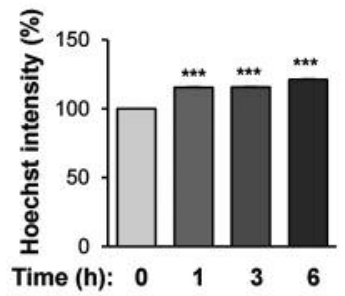

C

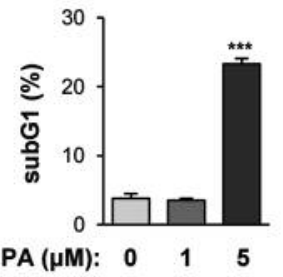

D

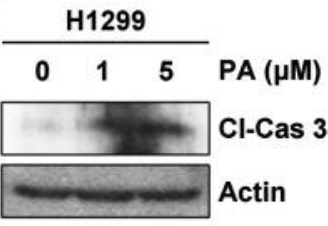

Figure 5. Induction of apoptotic cell death by papuamine treatment. (A, B) $H 1299$ cells were treated with papuamine for various time intervals. After staining cells with Hoechst 33342, cellular (A) and nuclear (B) morphological changes in papuamine-treated cells were analyzed using the Operetta High Content Imaging System. (C) The accumulation of cells in the sub-G1 phase of the cell cycle was determined by flow cytometry as described in the Materials and Methods section. (D) The expression of cleaved caspase 3 was determined by western blot analysis. PA: Papuamine. The bars represent the mean $\pm S D ; * p<0.05$, ** $p<0.01$, and $* * * p<0.001$, as determined by a two-sided Student's $t$-test.

Consistently, inhibitors of mitochondrial complex I, such as metformin and canagliflozin, exhibit promising anticancer effects in both in vitro and in vivo preclinical experiments; metformin has also been evaluated in clinical studies $(9,40-$ 42). These findings collectively suggest the promise of anticancer therapeutics targeting mitochondria. The discovery of additional mitochondria-targeting agents with diverse chemical entities is thus important to develop more effective anticancer drugs.

Natural products have been regarded as an important source for anticancer drug development $(43,44)$. Several chemotherapeutic agents, including paclitaxel, vinblastine, camptothecin, podophyllotoxin, and epothilone, have been

widely used in the clinic or have served as the chemical lead for further development of clinically available anticancer agents (45). In addition to plant-derived natural products, marine natural products are also attractive sources for drug development, and several marine natural products-derived anticancer compounds have been approved for clinical use (46-48). Indeed, several marine-derived cytotoxic and potential antitumor compounds have been identified in recent studies (49-51). In line with these reports, our results clearly suggest that papuamine is a novel marine-derived anticancer agent targeting mitochondria and thus may provide additional chemical entity to develop mitochondria-targeting anticancer agents in further studies.

The mechanism underlying papuamine-induced mitochondrial dysfunction and apoptotic cell death still needs to be determined, but some possible mechanisms of action were detailed here. First, as suggested previously, autophagy is one mechanism underlying papuamine-induced apoptosis (19). Our results revealed that papuamine elevated mitochondrial intensity but gradually decreased the number of mitochondria with normal morphology. These findings collectively suggest that papuamine may induce mitochondrial fission, and generate damaged, non-functional mitochondria, eventually leading to the induction of mitophagy, a cellular process that removes damaged mitochondria (52). However, previous reports have suggested that, similar to autophagy, mitophagy is an adaptive response to stress and protects against apoptosis $(53,54)$. Thus, the precise role of mitophagy in papuamine-induced apoptosis needs to be determined in future studies. In addition, it is possible that nitrogen-containing compounds, such as alkaloids (e.g., papuamine), can interact with proteins, modulating their activity and interactions with other proteins (55). These alterations might diminish the enzymatic activity of proteins involved in the cellular defense against oxidative stress, causing cellular damage including mitochondrial death and apoptotic cell death. Meanwhile, a recently published paper classified various mitochondria-targeting anticancer agents into nine categories, including hexokinase inhibitors, thiol redox inhibitors, and electron redox chain targeting drugs (56). Several anticancer alkaloids are also known to target mitochondria through inducing alterations in mitochondrial bioenergetics, metabolic pathways, and morphology (57). Previous reports have suggested that inhibition of oxidative phosphorylation leads to apoptosis by inducing mitochondrial ROS production $(58,59)$. In our results, papuamine reduced energy production, elevated mitochondrial ROS level, and induced apoptosis in NSCLC cells. Thus, disrupting mitochondria function through interacting with the mitochondria respiratory chain might be one of the mechanisms underlying papuamine-mediated apoptosis. This possibility should also be examined in future studies. 
In summary, the present study suggests that papuamine exerts its anticancer effects by causing mitochondrial dysfunction, thereby decreasing cellular energy production and inducing apoptosis. Although additional studies should examine the effect of papuamine on the proliferation of normal cells and evaluate the anticancer effect of papuamine in vivo, our results suggest that papuamine is a promising marine natural product-derived, mitochondria-targeting anticancer compound. Further studies are warranted to evaluate the anticancer effectiveness of papuamine in advanced preclinical and clinical settings.

\section{Conflicts of Interest}

The Authors have no conflicts of interest to declare regarding this study.

\section{Authors' Contributions}

Study concept and design: HY Lee. Acquisition of data: HY Min, Y Jung, KH Park. Analysis and interpretation of data: HY Min, Y Jung, KH Park. HY Lee. Drafting of the manuscript: HY Min, HY Lee. Study supervision: HY Lee.

\section{Acknowledgements}

This study was supported by grants from the National Research Foundation of Korea (NRF), the Ministry of Science and ICT (MSIT), Republic of Korea (No. NRF-2016R1A3B1908631) and the Ministry of Education, Republic of Korea (No. NRF2016R1A6A3A11935661).

\section{References}

1 Torre LA, Bray F, Siegel RL, Ferlay J, Lortet-Tieulent J and Jemal A: Global cancer statistics, 2012. CA Cancer J Clin 65(2): 87-108, 2015. PMID: 25651787. DOI: 10.3322/caac.21262

2 Siegel RL, Miller KD and Jemal A: Cancer statistics, 2017. CA Cancer J Clin 67(1): 7-30, 2017. PMID: 28055103. DOI: $10.3322 /$ caac. 21387

3 Heuvers ME, Hegmans JP, Stricker BH and Aerts JG: Improving lung cancer survival; time to move on. BMC Pulm Med 12: 77, 2012. PMID: 23234250. DOI: 10.1186/1471-2466-12-77

4 Chan BA and Hughes BG: Targeted therapy for non-small cell lung cancer: Current standards and the promise of the future Transl Lung Cancer Res 4(1): 36-54, 2015. PMID: 25806345 DOI: $10.3978 / \mathrm{j}$.issn.2218-6751.2014.05.01

5 Malhotra J, Jabbour SK and Aisner J: Current state of immunotherapy for non-small cell lung cancer. Transl Lung Cancer Res 6(2): 196-211, 2017. PMID: 28529902. DOI: $10.21037 /$ tlcr.2017.03.01

6 Spaans JN and Goss GD: Drug resistance to molecular targeted therapy and its consequences for treatment decisions in nonsmall-cell lung cancer. Front Oncol 4: 190, 2014. PMID: 25101246. DOI: 10.3389/fonc 2014.00190

7 Amaral $\mathrm{T}$ and Garbe C: Acquired resistance mechanisms to immunotherapy. Ann Transl Med 4(24): 547, 2016. PMID: 28149908. DOI: $10.21037 / \mathrm{atm} .2016 .12 .21$
8 Hanahan D and Weinberg RA: Hallmarks of cancer: The next generation. Cell 144(5): 646-674, 2011. PMID: 21376230. DOI: 10.1016/j.cell.2011.02.013

9 Fulda S, Galluzzi L and Kroemer G: Targeting mitochondria for cancer therapy. Nat Rev Drug Discov 9(6): 447-464, 2010. PMID: 20467424, DOI: 10.1038/nrd3137

10 Wallace DC: Mitochondria and cancer. Nat Rev Cancer 12(10): 685-698, 2012. PMID: 23001348. DOI: $10.1038 / \mathrm{nrc} 3365$

11 Phan LM, Yeung SC and Lee MH: Cancer metabolic reprogramming: Importance, main features, and potentials for precise targeted anti-cancer therapies. Cancer Biol Med 11(1): 1-19, 2014. PMID: 24738035. DOI: 10.7497/j.issn.20953941.2014.01.001

12 Assan R, Heuclin C, Girard JR, LeMaire F and Attali JR: Phenformin-induced lactic acidosis in diabetic patients. Diabetes 24(9): 791-800, 1975. PMID: 808437. DOI: 10.2337/diab.24.9.791

13 Caboni P, Sherer TB, Zhang N, Taylor G, Na HM, Greenamyre JT and Casida JE: Rotenone, deguelin, their metabolites, and the rat model of parkinson's disease. Chem Res Toxicol 17(11): 1540-1548, 2004. PMID: 15540952. DOI: 10.1021/tx049867r

14 Barrett AG, Boys ML and Boehm TL: Total synthesis of (+)papuamine: An antifungal pentacyclic alkaloid from a marine sponge, Haliclona sp. J Org Chem 61(2): 685-699, 1996. PMID: 11666992. DOI: $10.1021 /$ jo $951413 \mathrm{z}$

15 Farooqi AA, Fayyaz S, Hou MF, Li KT, Tang JY and Chang HW: Reactive oxygen species and autophagy modulation in nonmarine drugs and marine drugs. Mar Drugs 12(11): 5408-5424, 2014. PMID: 25402829, DOI: $10.3390 / \mathrm{md} 12115408$

16 Li Q, Chen C, Kapadia A, Zhou Q, Harper MK, Schaack J and LaBarbera DV: 3D models of epithelial-mesenchymal transition in breast cancer metastasis: High-throughput screening assay development, validation, and pilot screen. J Biomol Screen 16(2): 141-154, 2011. PMID: 21297102. DOI: 10.1177/ 1087057110392995

17 Kanno SI, Yomogida S, Tomizawa A, Yamazaki H, Ukai K, Mangindaan RE, Namikoshi $\mathrm{M}$ and Ishikawa M: Combined effect of papuamine and doxorubicin in human breast cancer mcf-7 cells. Oncol Lett 8(2): 547-550, 2014. PMID: 25013468. DOI: $10.3892 / \mathrm{ol} .2014 .2218$

18 Bae W, Lim HK, Kim KM, Cho H, Lee SY, Jeong C-S, Lee H$\mathrm{S}$ and Jung J: Apoptosis-inducing activity of marine sponge haliclona sp. Extracts collected from kosrae in nonsmall cell lung cancer A549 cells. Evid Based Complement Alternat Med 2015: 717959, 2015. PMID: 26236382. DOI: 10.1155/ 2015/717959

19 Kanno S, Yomogida S, Tomizawa A, Yamazaki H, Ukai K, Mangindaan RE, Namikoshi $\mathrm{M}$ and Ishikawa M: Papuamine causes autophagy following the reduction of cell survival through mitochondrial damage and JNK activation in MCF-7 human breast cancer cells. Int J Oncol 43(5): 1413-1419, 2013. PMID: 24026338. DOI: 10.3892/ijo.2013.2093

20 Schneider CA, Rasband WS and Eliceiri KW: NIH image to imageJ: 25 years of image analysis. Nat Methods 9(7):671-675, 2012. PMID: 22930834. DOI: 10.1038/nmeth.2089

21 Weinberg SE and Chandel NS: Targeting mitochondria metabolism for cancer therapy. Nat Chem Biol 11(1): 9-15, 2015. PMID: 25517383. DOI: $10.1038 /$ nchembio. 1712

22 Jang M, Kim SS and Lee J: Cancer cell metabolism: Implications for therapeutic targets. Exp Mol Med 45(10): e45, 2013. PMID: 24091747. DOI: 10.1038/emm.2013.85 
23 Bonora M, Patergnani S, Rimessi A, De Marchi E, Suski JM, Bononi A, Giorgi C, Marchi S, Missiroli S, Poletti F, Wieckowski MR and Pinton P: Atp synthesis and storage. Purinergic Signal 8(3): 343-357, 2012. PMID: 22528680. DOI: $10.1007 / \mathrm{s} 11302-012-9305-8$

24 Hardie DG, Ross FA and Hawley SA: Ampk: A nutrient and energy sensor that maintains energy homeostasis. Nat Rev Mol Cell Biol 13(4): 251-262, 2012. PMID: 22436748. DOI:10.1038/nrm3311

25 Vizler C, Nagy T, Kusz E, Glavinas H and Duda E: Flow cytometric cytotoxicity assay for measuring mammalian and avian NK cell activity. Cytometry 47(3): 158-162, 2002. PMID: 11891720. DOI: $10.1002 /$ cyto. 10066

26 Nieminen AL, Byrne AM, Herman B and Lemasters JJ: Mitochondrial permeability transition in hepatocytes induced by t-BuOOH: NAD $(\mathrm{P}) \mathrm{H}$ and reactive oxygen species. Am J Physiol 272(4 Pt 1): C1286-1294, 1997. PMID: 9142854. DOI: 10.1152/ajpcell.1997.272.4.C1286

27 Robinson KM, Janes MS, Pehar M, Monette JS, Ross MF, Hagen TM, Murphy MP and Beckman JS: Selective fluorescent imaging of superoxide in vivo using ethidium-based probes. Proc Natl Acad Sci USA 103(41): 15038-15043, 2006. PMID: 17015830. DOI: 10.1073/pnas.0601945103

28 Zou C, Wang Y and Shen Z: 2-NBDG as a fluorescent indicator for direct glucose uptake measurement. J Biochem Biophys Methods 64(3): 207-215, 2005. PMID: 16182371. DOI: 10.1016/j.jbbm.2005.08.001

29 Huet O, Petit JM, Ratinaud MH and Julien R: NADH-dependent dehydrogenase activity estimation by flow cytometric analysis of 3-(4,5-dimethylthiazolyl-2-yl)-2,5-diphenyltetrazolium bromide (MTT) reduction. Cytometry 13(5): 532-539, 1992. PMID: 1633732. DOI: $10.1002 /$ cyto. 990130513

30 Bortner CD and Cidlowski JA: A necessary role for cell shrinkage in apoptosis. Biochem Pharmacol 56(12): 1549-1559, 1998. PMID: 9973175. DOI: 10.1016/s0006-2952(98)00225-1

31 Tone S, Sugimoto K, Tanda K, Suda T, Uehira K, Kanouchi H, Samejima K, Minatogawa Y and Earnshaw WC: Three distinct stages of apoptotic nuclear condensation revealed by time-lapse imaging, biochemical and electron microscopy analysis of cellfree apoptosis. Exp Cell Res 313(16): 3635-3644, 2007. PMID: 17643424. DOI: $10.1016 /$ j.yexcr.2007.06.018

32 Min HY and Lee HY: Oncogene-driven metabolic alterations in cancer. Biomol Ther (Seoul) 26(1): 45-56, 2018. PMID: 29212306. DOI: $10.4062 /$ biomolther.2017.211

33 Zhao Y, Butler EB and Tan M: Targeting cellular metabolism to improve cancer therapeutics. Cell Death Dis 4(3): e532, 2013. PMID: 23470539. DOI: $10.1038 /$ cddis.2013.60

34 Iommarini L, Ghelli A, Gasparre G and Porcelli AM: Mitochondrial metabolism and energy sensing in tumor progression. Biochim Biophys Acta 1858(8): 582-590, 2017. PMID: 28213331. DOI: 10.1016/j.bbabio.2017.02.006

35 He H, Li DW, Yang LY, Fu L, Zhu XJ, Wong WK, Jiang FL and Liu Y: A novel bifunctional mitochondria-targeted anticancer agent with high selectivity for cancer cells. Sci Rep 5: 13543, 2015. PMID: 26337336. DOI: 10.1038/srep13543

36 Zhang X, Zhang S, Zhu S, Chen S, Han J, Gao K, Zeng JZ and Yan X: Identification of mitochondria-targeting anticancer compounds by an in vitro strategy. Anal Chem 86(11): 52325237, 2014. PMID: 24823627. DOI: 10.1021/ac500918g

37 Liu Y, Zhang X, Zhou M, Nan X, Chen X and Zhang X: Mitochondrial-targeting lonidamine-doxorubicin nanoparticles for synergistic chemotherapy to conquer drug resistance. ACS Appl Mater Interfaces 9(50): 43498-43507, 2017. PMID: 29171954. DOI: $10.1021 /$ acsami.7b14577

38 Zheng J: Energy metabolism of cancer: Glycolysis versus oxidative phosphorylation (review). Oncol Lett 4(6): 1151-1157, 2012. PMID: 23226794. DOI: 10.3892/ol.2012.928

$39 \mathrm{Zu}$ XL and Guppy M: Cancer metabolism: Facts, fantasy, and fiction. Biochem Biophys Res Commun 313(3): 459-465, 2004. PMID: 14697210. DOI: 10.1016/j.bbrc.2003.11.136

40 Wheaton WW, Weinberg SE, Hamanaka RB, Soberanes S, Sullivan LB, Anso E, Glasauer A, Dufour E, Mutlu GM, Budigner GS and Chandel NS: Metformin inhibits mitochondrial complex I of cancer cells to reduce tumorigenesis. Elife 3: e02242, 2014. PMID: 24843020. DOI: 10.7554/eLife.02242

41 Urra FA, Munoz F, Lovy A and Cardenas C: The mitochondrial complex(I)ty of cancer. Front Oncol 7: 118, 2017. PMID: 28642839. DOI: $10.3389 /$ fonc 2017.00118

42 Villani LA, Smith BK, Marcinko K, Ford RJ, Broadfield LA, Green AE, Houde VP, Muti P, Tsakiridis T and Steinberg GR: The diabetes medication canagliflozin reduces cancer cell proliferation by inhibiting mitochondrial complex-I supported respiration. Mol Metab 5(10): 1048-1056, 2016. PMID: 27689018. DOI: 10.1016/j.molmet.2016.08.014

43 Newman DJ and Cragg GM: Natural products as sources of new drugs over the 30 years from 1981 to 2010. J Nat Prod 75(3): 311-335, 2012. PMID: 22316239. DOI: 10.1021/np200906s

44 Kinghorn AD, EJ DEB, Lucas DM, Rakotondraibe HL, Orjala J, Soejarto DD, Oberlies NH, Pearce CJ, Wani MC, Stockwell BR, Burdette JE, Swanson SM, Fuchs JR, Phelps MA, Xu L, Zhang $\mathrm{X}$ and Shen YY: Discovery of anticancer agents of diverse natural origin. Anticancer Res 36(11): 5623-5637, 2016. PMID: 27793884. DOI: 10.21873/anticanres.11146

45 Mann J: Natural products in cancer chemotherapy: Past, present and future. Nat Rev Cancer 2(2): 143-148, 2002. PMID: 12635177. DOI: $10.1038 /$ nrc723

46 Molinski TF, Dalisay DS, Lievens SL and Saludes JP: Drug development from marine natural products. Nat Rev Drug Discov 8(1): 69-85, 2009. PMID: 19096380. DOI: 10.1038/ $\operatorname{nrd} 2487$

47 Newman DJ and Cragg GM: Marine natural products and related compounds in clinical and advanced preclinical trials. J Nat Prod 67(8): 1216-1238, 2004. PMID: 15332835. DOI: 10.1021/ np040031y

48 Yun CW, Kim HJ and Lee SH: Therapeutic application of diverse marine-derived natural products in cancer therapy. Anticancer Res 39(10): 5261-5284, 2019. PMID: 31570422. DOI: 10.21873 /anticanres.13721

49 Liu R, Zhu T, Li D, Gu J, Xia W, Fang Y, Liu H, Zhu W and Gu Q: Two indolocarbazole alkaloids with apoptosis activity from a marine-derived Actinomycete Z(2)039-2. Arch Pharm Res 30(3): 270-274, 2007. PMID: 17424930. DOI: 10.1007/bf02977605

50 Ren H and Liu W-W: Nidurufin as a new cell cycle inhibitor from marine-derived fungus Penicillium Flavidorsum SHK1-27. Arch Pharm Res 34(6): 901-905, 2011. PMID: 21725810. DOI: 10.1007/s12272-011-0606-1

51 Zhuravleva OI, Afiyatullov SS, Vishchuk OS, Denisenko VA, Slinkina NN and Smetanina OF: Decumbenone C, a new cytotoxic decaline derivative from the marine fungus Aspergillus Sulphureus KMM 4640. Arch Pharm Res 35(10): 1757-1762, 2012. PMID: 23139126. DOI: 10.1007/s12272-012-1007-9 
52 Ding WX and Yin XM: Mitophagy: Mechanisms, pathophysiological roles, and analysis. Biol Chem 393(7): 547-564, 2012. PMID: 22944659. DOI: 10.1515/hsz-2012-0119

53 Kubli DA and Gustafsson AB: Mitochondria and mitophagy: The yin and yang of cell death control. Circ Res 111(9): 12081221, 2012. PMID: 23065344. DOI: 10.1161/CIRCRESAHA. 112.265819

54 Zhang HT, Mi L, Wang T, Yuan L, Li XH, Dong LS, Zhao P, Fu JL, Yao BY and Zhou ZC: PINK1/Parkin-mediated mitophagy play a protective role in manganese induced apoptosis in $\mathrm{SH}$ SY5Y cells. Toxicol In Vitro 34: 212-219, 2016. PMID: 27091500. DOI: $10.1016 /$ j.tiv.2016.04.006

55 Wink M: Molecular modes of action of cytotoxic alkaloids: From DNA intercalation, spindle poisoning, topoisomerase inhibition to apoptosis and multiple drug resistance. Alkaloids Chem Biol 64: 1-47, 2007. PMID: 18085328. DOI: 10.1016/ s1099-4831(07)64001-2

56 Neuzil J, Dong LF, Rohlena J, Truksa J and Ralph SJ: Classification of mitocans, anti-cancer drugs acting on mitochondria. Mitochondrion 13(3): 199-208, 2013. PMID: 22846431. DOI: $10.1016 /$ j.mito.2012.07.112
57 Urra FA, Cordova-Delgado M, Pessoa-Mahana H, RamirezRodriguez O, Weiss-Lopez B, Ferreira J and Araya-Maturana R: Mitochondria: A promising target for anticancer alkaloids. Curr Top Med Chem 13(17): 2171-2183, 2013. PMID: 23978135. DOI: $10.2174 / 15680266113139990150$

58 Li N, Ragheb K, Lawler G, Sturgis J, Rajwa B, Melendez JA and Robinson JP: Mitochondrial complex i inhibitor rotenone induces apoptosis through enhancing mitochondrial reactive oxygen species production. J Biol Chem 278(10): 8516-8525, 2003. PMID: 12496265 . DOI: $10.1074 /$ jbc.M210432200

59 Dong LF, Low P, Dyason JC, Wang XF, Prochazka L, Witting PK, Freeman R, Swettenham E, Valis K, Liu J, Zobalova R, Turanek J, Spitz DR, Domann FE, Scheffler IE, Ralph SJ and Neuzil J: Alpha-tocopheryl succinate induces apoptosis by targeting ubiquinone-binding sites in mitochondrial respiratory complex II. Oncogene 27(31): 4324-4335, 2008. PMID: 18372923. DOI: $10.1038 /$ onc. 2008.6

Received November 13, 2019

Revised November 23, 2019

Accepted November 28, 2019 